\title{
Workplace smoking policies in the United States: results from a national survey of more than 100000 workers
}

\author{
Karen K Gerlach, Donald R Shopland, Anne M Hartman, James T Gibson, \\ Terry F Pechacek
}

\begin{abstract}
Objective-To determine the prevalence of smoking policies in indoor work environments as reported by a nationally representative sample of workers in the United States.
\end{abstract}

Design-Cross-sectional survey of households within the United States.

Setting-All 50 states and the District of Columbia, 1992-93.

Participants-Currently employed indoor workers 15 years of age and older who responded to the National Cancer Institute's Tobacco Use Supplement to the Current Population Survey $(n=100$ 561). Main outcome measures-The prevalence and restrictiveness of workplace smoking policies as reported by workers currently employed in indoor workplaces in the United States.

Results-Most of the indoor workers surveyed $(81.6 \%)$ reported that their place of work had an official policy that addressed smoking in the workplace; $46.0 \%$ reported that their workplace policy did not permit smoking in either the public/common areas-for example, restrooms and cafeterias-or the work areas of the workplace. The reporting of these "smoke-free" policies varied significantly by gender, age, race/ethnicity, smoking status, and occupation of the worker.

Conclusions-Although nearly half of all indoor workers in this survey reported that they had a smoke-free policy in their workplace, significant numbers of workers, especially those in blue-collar and service occupations, reported smoke-free rates well below the national average. If implemented, the US Occupational Safety and Health Administration's proposed regulation to require worksites to be smoke-free has the potential to increase significantly the percentage of American workers covered by these policies and to eliminate most of the disparity currently found across occupational groups.

\section{(Tobacco Control 1997;6:199-206)}

Keywords: workplace smoking policy; smoke-free worksites; United States

\section{Introduction}

The possibility that ambient tobacco smoke could harm non-smokers was first articulated in January 1971 by then US Surgeon General
Jesse L Steinfeld, who called for a national $\vec{\omega}$ "Nonsmokers' Bill of Rights." Twenty years $\stackrel{\odot}{\circ}$ later Dr Steinfeld reflected, "No other action or suggestion regarding cigarette use had elicited such a torrent of mail as the call for a $\mathscr{E}$ nonsmokers' bill of rights. The tally [of 음 responses] was almost 20 to 1 in favor of the proposal."2 Steinfeld also directed the National $\mathbb{D}$ Clearinghouse for Smoking and Health (predecessor to the Office on Smoking and Health) $\stackrel{\mathbb{D}}{3}$ to include in the next report of the Surgeon $\underset{\mathbb{D}}{ }$ General a review of the available scientific evidence on the health effects of environmental tobacco smoke (ETS). ${ }^{3}$

In 1986 reports from both the US Surgeon General and the National Academy of Sciences again addressed the hazardous nature of ETS and concluded that ETS was a major health

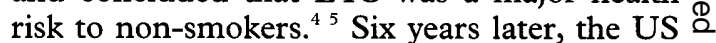
Environmental Protection Agency (EPA) $\overrightarrow{\overrightarrow{0}}$ issued a landmark report that further detailed 3 the health hazards of ETS, classifying it as a group A (known human) carcinogen and estimating that each year 3000 cases of lung cancer in non-smokers were attributable to ETS exposure. ${ }^{6}$ Reflecting the growing o evidence that ETS poses a health risk, the US Public Health Service included in its national health goals that $75 \%$ of worksites either prohibit or severely restrict smoking by the year $2000 .^{7}$

This report presents a comprehensive estimate of the number of American workers covered by official workplace smoking policies based on a survey of currently employed workers. The Current Population Survey (CPS), which has been in existence since 1947, is o designed to obtain labour force indicators for $N$ the US Bureau of Labor Statistics, and, as such, provides a good vehicle for obtaining 0 workplace-related information from American 0 workers. In 1992 and 1993 the National Can- $\frac{D}{D}$ cer Institute (NCI) appended to the CPS a $\stackrel{?}{+}$ Tobacco Use Supplement, which assessed, $\square$ among other things, the presence and $\stackrel{+}{\mathbb{D}}$ restrictiveness of workplace smoking policies. ? The data from this survey, obtained from $\stackrel{\unrhd}{\unrhd}$ interviews with more than 100000 workers, are presented by age, gender, race/ethnicity, 8 and occupational groups.

\section{Methods}

The CPS is a continuous monthly survey, which focuses on labour force indicators for the civilian, non-institutionalised American 
population aged 15 years and older. In 1992, a 40-item Tobacco Use Supplement was developed by NCI staff and pre-tested by trained Census Bureau interviewers before full field implementation. For these analyses, "non-smoker" refers to both former and neversmokers. Questions on smoking and tobacco use and the presence and characteristics of workplace smoking policies were added to the CPS for the September 1992, January 1993, and May 1993 surveys. The questions used on this survey to determine smoking status were identical to those adopted in 1992 by the National Center for Health Statistics and currently in use on the National Health Interview Survey. ${ }^{8}$

The complete CPS methodology has been published in detail elsewhere. ${ }^{9}$ Briefly, the CPS is a probability sample based on a stratified sampling scheme of clusters of four neighbouring households. The three main sources are households listed in the most recent decennial census (for the 1992-93 surveys the 1980 Census base was in use), updated building permits, and area sampling when no Bureau of the Census address lists exist. The 1985-94 sample, drawn from 729 areas comprising 1973 counties and independent cities, covered every state and the District of Columbia. Approximately 59000 housing units or living quarters were assigned for interview, and about 56000 households containing approximately 110000 persons were interviewed each month. All strata were defined within state boundaries, and the sample was allocated among the states to produce state, Census region and division, and national labour force estimates, while keeping the total sample size to a minimum.

Most interviews for the CPS are conducted with a knowledgeable household representative who responds for all eligible household members. Typically, slightly more than half of all interviews are self-respondents; the remainder are by proxy. ${ }^{10}$ An attempt was made to minimise the proportion of proxy responses to the 1992-93 Tobacco Use Supplement because the answers to many questions of interest to the NCI would be known only by the person to be interviewed. Thus, the Supplement questionnaire was administered "off document" on a form separate from the standard labour force core questionnaire, so that data collection was not constrained by the one week limit for labour statistics collection. This procedure reduced proxy responses to $18 \%$. Only self-responses were used for this analysis.

Response rates to the CPS labour force core questionnaire were $95.7 \%, 95.3 \%$, and $95.2 \%$ for September 1992, January 1993, and May 1993, respectively. The corresponding response rates for the Tobacco Use Supplement of those responding to the CPS core were $88.5 \%, 89.1 \%$, and $86.1 \%$, respectively. Approximately $25 \%$ of all interviews were conducted in person and $75 \%$ by telephone. For Spanish-language interviews, a fully translated Tobacco Use Supplement was used. For interviews in all other languages, a Census Bureau employee fluent in the required language translated the questionnaire. When no Census employee could speak the language required, either a community or household member served as the translator. In all cases for which separate estimates are given for whites and blacks, the data for Hispanics have been excluded.

\section{OCCUPATION AND WORKPLACE DEFINITIONS}

Routine labour force questions from the CPS core were used to determine each respondent's employment status (including those who were self-employed) and to classify each worker by a standard occupational group. In the CPS, there are 500 categories for employed persons; Census aggregates these categories into 45 groups. This classification system uses a three digit code (000-905) developed from the 1980 Standard Occupational Classification. ${ }^{11} \mathrm{Be}-$ cause NCI was interested primarily in measuring the extent to which indoor workers were covered by official workplace smoking policies, additional questions from the Tobacco Use Supplement were used to identify eligible employees. To be included in the analysis, an individual had to have been (a) 15 years of age or older at the time of interview; (b) currently employed outside the home but not self-employed; (c) not working outdoors or in a motor vehicle; (d) not travelling to different buildings or sites; and (e) not working in someone else's home. These analyses used the Census Bureau definition of "worker", which is a person who worked in the past week or who would have worked in the past week (that is, had a job) but did not work because of illness, vacation, or labour dispute. Both fulltime and parttime workers were included in these analyses.

WORKPLACE SMOKING POLICY DEFINITIONS

All eligible respondents were queried: "Does your place of work have an official policy that restricts smoking in any way?" Those who responded "yes" were further asked: "Which of these best describes your place of work's smoking policy for indoor public or common areas, such as lobbies, rest rooms and lunch rooms?" and "Which of these best describes your place of work's smoking policy for work areas?" Response choices for each were: "Not allowed in any areas ....", "Allowed in some ...", or "Allowed in all ...".

According to this algorithm, the most restrictive policies were those in which the worker reported an official workplace policy that did not allow smoking in any public or common areas of the workplace or in the work areas of the workplace. In the tables, this policy is designated "smoke-free". The policy indicated by "No smoking, work areas only" describes the situation in which a worker reported that smoking was allowed in some or all public or common areas but not in the work area of his or her workplace. All other combinations of responses that permitted smoking in some or all work areas are labelled "Smoking allowed". Those workers responding "Don't know" ( $n=1830,1.9 \%)$ to any of 
the above questions were excluded from the analyses. The remaining respondents $(n=$ 100561 ) included 41765 men and 58796 women.

Statistical analyses were performed using SAS version 6.11. ${ }^{12}$ Supplement weights, adjusting for overall Supplement non-response and Supplement self-response only, were produced using a special algorithm developed by the Bureau of the Census. Only the self-response weights were used in these analyses because work policy-related questions were asked only of self-respondents. Standard errors, which were used in computing the $95 \%$ confidence intervals (CIs), were produced using the CPS design effect adjustments developed by the Bureau of the Census. ${ }^{11}$

\section{Results}

Overall, $81.6 \%$ of the indoor workers surveyed reported that their employer had an official workplace smoking policy that restricted smoking in some way (table 1). On average, men were approximately $5 \%$ less likely than women to report that their employer had implemented a smoking policy $(P<0.0001)$. Among the four major racial/ethnic groups, Asian/Pacific Islanders were the most likely, and Hispanics were the least likely, to report that their employer had an official policy that restricted smoking.

Although more than $80 \%$ of workers were covered by an official workplace policy, slightly less than half of those surveyed reported that they were covered by a policy that prohibited smoking in both the work and public/common areas of the workplace ("smoke-free" workplaces). Significant differences were also observed between the sexes with respect to these smoke-free policies: $51.0 \%$ of women but only $39.7 \%$ of men reported working under these policies $(\mathrm{P}<0.0001)$. The analysis showed a consistent $10-12$ percentage point difference between men and women, and this pattern was observed for each of the four major racial/ethnic groups (table 1). An equal percentage $(18 \%)$ of both men and women reported that their employer had a policy that prohibited smoking in the work area only.

Black workers had relatively high rates of $ᄋ$ working for employers who had implemented an official policy, but they were less likely than whites and Asian/Pacific Islanders to work $\overrightarrow{\bar{N}}$ under a smoke-free policy. This pattern was $\overrightarrow{0}$ particularly evident among black men among $\frac{\overline{0}}{\sigma}$ whom slightly more than a third reported $\frac{\bar{\sigma}}{7}$ working in a smoke-free workplace. The $\mathbb{\Phi}$ responses of women of Asian/Pacific Islander ancestry showed the highest prevalence of such workplace policies $(57.2 \%)$

Among the American workers in this survey, $\overrightarrow{\vec{\omega}}$ significantly more reported that smoking was not permitted in their workplace than reported that some smoking was allowed in either the $\omega$ work or public/common areas of their work- $\vec{\theta}$ place $(46.0 \%$ vs $35.7 \%)$ ( $\mathrm{P}<0.0001)$. Nearly two-thirds of all workers reported that smoking was not permitted within their work area.

Table 2 presents data on workplace smoking $\mathbb{D}$ policies and the restrictiveness of those policies by gender and age of the worker. These data clearly indicate a significant age disparity in the distribution of workplace smoking policies. $\overrightarrow{0}$ Those American workers in the youngest age categories (15-19 and 20-24 years) were the least likely to report that they had any policy, and they were the least likely to work under a smoke-free policy. Only $32.1 \%$ of workers aged 15-19 were working under a smoke-free policy, and only $41 \%$ of $20-24$ year olds were covered $\overrightarrow{\vec{P}}$ by such a policy. Compared with men, women in every age group reported higher rates of working in smoke-free workplaces $(P<0.0001)$.

Except for workers aged 55 and older, the percentages of both male and female workers reporting the presence of both an official policy and a policy that prohibited workplace smoking increased with age. The older workers were somewhat less likely than those aged 35-44 and 45-54 to report being covered by more restrictive policies; however, these differences were not as striking as those between older workers and those younger than age 25

Table 1 American workers ${ }^{*}$ covered by various workplace smoking policies, by race and gender

\begin{tabular}{|c|c|c|c|c|c|c|c|c|}
\hline Racet/gender & $\begin{array}{l}\text { Smoke-free } \\
(\%)\end{array}$ & $(95 \% C I)$ & $\begin{array}{l}\text { No policy } \\
(\%)\end{array}$ & $(95 \% C I)$ & $\begin{array}{l}\text { No smoking, work } \\
\text { areas only } \\
(\%)\end{array}$ & $(95 \% C I)$ & $\begin{array}{l}\text { Smoking } \\
\text { allowed } \\
(\%)\end{array}$ & $(95 \% C I)$ \\
\hline $\begin{array}{l}\text { Total } \\
\text { Male } \\
\text { Female }\end{array}$ & $\begin{array}{l}46.0 \\
39.7 \\
51.0\end{array}$ & $\begin{array}{l}(45.7-46.3) \\
(39.2-40.2) \\
(50.5-51.5)\end{array}$ & $\begin{array}{l}18.4 \\
20.9 \\
16.3\end{array}$ & $\begin{array}{l}(18.0-18.8) \\
(20.5-21.4) \\
(15.9-16.7)\end{array}$ & $\begin{array}{l}18.7 \\
18.8 \\
18.6\end{array}$ & $\begin{array}{l}(18.4-19.0) \\
(18.4-19.2) \\
(18.2-19.0)\end{array}$ & $\begin{array}{l}17.0 \\
20.6 \\
14.1\end{array}$ & $\begin{array}{l}(16.7-17.3) \\
(20.1-21.1) \\
(13.8-14.5)\end{array}$ \\
\hline $\begin{array}{l}\text { White } \\
\text { Male } \\
\text { Female }\end{array}$ & $\begin{array}{l}46.2 \\
40.0 \\
51.4\end{array}$ & $\begin{array}{l}(45.8-46.6) \\
(39.4-40.6) \\
(50.8-52.0)\end{array}$ & $\begin{array}{l}18.5 \\
20.8 \\
16.6\end{array}$ & $\begin{array}{l}(18.2-18.8) \\
(20.3-21.3) \\
(16.2-17.0)\end{array}$ & $\begin{array}{l}18.2 \\
18.4 \\
18.1\end{array}$ & $\begin{array}{l}(17.9-18.5) \\
(17.9-18.9) \\
(17.7-18.5)\end{array}$ & $\begin{array}{l}17.1 \\
20.9 \\
14.0\end{array}$ & $\begin{array}{l}(16.8-17.4) \\
(20.4-21.4) \\
(13.6-14.4)\end{array}$ \\
\hline $\begin{array}{l}\text { Black } \\
\text { Male } \\
\text { Female }\end{array}$ & $\begin{array}{l}43.3 \\
36.4 \\
47.7\end{array}$ & $\begin{array}{l}(42.2-44.4) \\
(34.7-38.2) \\
(46.3-49.1)\end{array}$ & $\begin{array}{l}16.5 \\
19.8 \\
14.5\end{array}$ & $\begin{array}{l}(15.7-17.3) \\
(18.4-21.3) \\
(13.5-15.5)\end{array}$ & $\begin{array}{l}22.7 \\
22.9 \\
22.6\end{array}$ & $\begin{array}{l}(21.8-23.6) \\
(21.4-24.4) \\
(21.4-23.8)\end{array}$ & $\begin{array}{l}17.4 \\
20.9 \\
15.2\end{array}$ & $\begin{array}{l}(16.6-18.3) \\
(19.4-22.4) \\
(14.2-16.2)\end{array}$ \\
\hline $\begin{array}{l}\text { Hispanic } \\
\text { Male } \\
\text { Female }\end{array}$ & $\begin{array}{l}45.1 \\
38.9 \\
51.0\end{array}$ & $\begin{array}{l}(43.3-46.9) \\
(36.4-41.5) \\
(48.5-53.5)\end{array}$ & $\begin{array}{l}21.2 \\
25.1 \\
17.6\end{array}$ & $\begin{array}{l}(19.7-22.7) \\
(22.8-27.4) \\
(15.7-19.5)\end{array}$ & $\begin{array}{l}17.6 \\
17.7 \\
17.5\end{array}$ & $\begin{array}{l}(16.2-19.0) \\
(15.7-19.7) \\
(15.6-19.4)\end{array}$ & $\begin{array}{l}16.1 \\
18.3 \\
14.0\end{array}$ & $\begin{array}{l}(14.8-17.4) \\
(16.3-20.3) \\
(12.2-15.8)\end{array}$ \\
\hline $\begin{array}{l}\text { Asian/Pacific } \\
\text { Islander } \\
\text { Male } \\
\text { Female }\end{array}$ & $\begin{array}{l}51.4 \\
46.1 \\
57.2\end{array}$ & $\begin{array}{l}(49.1-53.7) \\
(42.9-49.3) \\
(53.9-60.5)\end{array}$ & $\begin{array}{l}15.0 \\
\\
16.5 \\
13.5\end{array}$ & $\begin{array}{l}(13.4-16.6) \\
(14.1-18.9) \\
(11.2-15.8)\end{array}$ & $\begin{array}{l}18.9 \\
\\
19.9 \\
17.8\end{array}$ & $\begin{array}{l}(17.1-20.7) \\
(17.4-22.4) \\
(15.3-20.3)\end{array}$ & $\begin{array}{l}17.5 \\
11.6\end{array}$ & $\begin{array}{r}(13.1-16.3) \\
(15.1-19.9) \\
(9.5-13.7)\end{array}$ \\
\hline
\end{tabular}


Table 2 American workers* covered by various smoking policies, by age and gender

\begin{tabular}{|c|c|c|c|c|c|c|c|c|}
\hline Age and gender & $\begin{array}{l}\text { Smoke-free } \\
(\%)\end{array}$ & $(95 \% C I)$ & $\begin{array}{l}\text { No policy } \\
(\%)\end{array}$ & $(95 \% C I)$ & $\begin{array}{l}\text { No smoking, work } \\
\text { areas only } \\
(\%)\end{array}$ & $(95 \% C I)$ & $\begin{array}{l}\text { Smoking } \\
\text { allowed } \\
(\%)\end{array}$ & $(95 \% C I)$ \\
\hline Total, age $\geqslant 15$ & 46.0 & $(45.6-46.4)$ & 18.4 & $(18.1-18.7)$ & 18.7 & $(18.4-19.0)$ & 17.0 & $(16.7-17.3)$ \\
\hline $15-19$ & 32.1 & $(30.5-33.8)$ & 27.3 & $(25.7-28.9)$ & 23.3 & $(21.8-24.8)$ & 17.3 & $(16.0-18.6)$ \\
\hline $20-24$ & 40.9 & $(39.8-42.0)$ & 23.0 & $(22.1-23.9)$ & 19.6 & $(18.7-20.5)$ & 16.5 & $(15.7-17.3)$ \\
\hline $25-34$ & 45.0 & $(44.3-45.7)$ & 18.7 & $(18.2-19.2)$ & 18.8 & $(18.3-19.3)$ & 17.4 & (16.9-17.9) \\
\hline $35-44$ & 49.0 & $(48.3-49.7)$ & 16.3 & $(15.8-16.8)$ & 17.8 & $(17.3-18.4)$ & 17.0 & (16.5-17.5) \\
\hline $45-54$ & 49.5 & $(48.6-50.4)$ & 15.4 & $(14.8-16.0)$ & 18.4 & $(17.7-19.1)$ & 16.8 & $(16.2-17.5)$ \\
\hline $55+$ & 46.0 & $(44.9-47.1)$ & 19.1 & $(18.2-20.0)$ & 18.2 & $(17.3-19.1)$ & 16.7 & $(15.8-17.6)$ \\
\hline Males, age $\geqslant 15$ & 39.7 & $(39.2-40.2)$ & 20.9 & $(20.5-21.4)$ & 18.8 & $(18.4-19.2)$ & 20.6 & $(20.2-21.1)$ \\
\hline $15-19$ & 28.5 & $(26.2-30.9)$ & 27.2 & $(24.9-29.5)$ & 25.4 & $(23.1-27.7)$ & 18.9 & $(16.9-20.9)$ \\
\hline $20-24$ & 34.2 & $(32.6-35.8)$ & 25.7 & $(24.3-27.2)$ & 20.7 & $(19.4-22.1)$ & 19.4 & (18.1-20.7) \\
\hline $25-34$ & 38.5 & $(37.5-39.5)$ & 21.5 & $(20.7-22.3)$ & 19.3 & $(18.5-20.1)$ & 20.7 & $(19.9-21.5)$ \\
\hline $35-44$ & 42.2 & $(41.2-43.3)$ & 19.1 & $(18.3-19.9)$ & 17.6 & $(16.8-18.4)$ & 21.1 & $(20.2-22.0)$ \\
\hline $45-54$ & 44.0 & $(42.7-45.3)$ & 17.3 & $(16.3-18.3)$ & 17.8 & $(16.8-18.8)$ & 20.8 & $(19.7-21.9)$ \\
\hline $55+$ & 40.5 & $(38.8-42.2)$ & 22.1 & $(20.7-23.5)$ & 16.9 & $(15.6-18.2)$ & 20.6 & $(19.2-22.0)$ \\
\hline Females, age $\geqslant 15$ & 51.0 & $(50.5-51.5)$ & 16.3 & $(15.9-16.7)$ & 18.6 & $(18.2-19.0)$ & 14.1 & $(13.8-14.5)$ \\
\hline $15-19$ & 35.2 & $(32.9-37.5)$ & 27.4 & $(25.3-29.5)$ & 21.5 & $(19.5-23.5)$ & 15.9 & $(14.1-17.7)$ \\
\hline $20-24$ & 46.1 & $(44.6-47.6)$ & 21.0 & $(19.8-22.2)$ & 18.7 & $(17.6-19.8)$ & 14.3 & $(13.3-15.3)$ \\
\hline $25-34$ & 50.6 & $(49.7-51.5)$ & 16.3 & $(15.6-17.0)$ & 18.5 & $(17.8-19.2)$ & 14.6 & $(13.9-15.3)$ \\
\hline $35-44$ & 54.5 & $(53.5-55.5)$ & 14.0 & $(13.3-14.7)$ & 17.9 & $(17.2-18.6)$ & 13.6 & $(12.9-14.3)$ \\
\hline $45-54$ & 53.7 & $(52.5-54.9)$ & 13.9 & $(13.1-14.7)$ & 18.8 & $(17.9-19.7)$ & 13.7 & $(12.9-14.5)$ \\
\hline $55+$ & 50.4 & $(48.9-51.9)$ & 16.7 & $(15.6-17.8)$ & 19.2 & $(18.0-20.4)$ & 13.7 & (12.7-14.8) \\
\hline
\end{tabular}

^Smokers and non-smokers.

$\mathrm{CI}=$ confidence intervals.

Younger employees were also more likely than older employees to report that smoking was allowed in some public or common areas of the workplace but not in the work area.

Table 3 presents data on workplace smoking policy differences for currently employed workers classified as white collar (for example, professionals and management-level workers), service (such as hotel, restaurant, and janitorial workers) and blue collar (for example, skilled and unskilled labourers). A clear majority of all white-collar workers reported that their employer had instituted a smoke-free workplace policy. White-collar employees were more than one and a half times as likely as service workers and nearly twice as likely as blue-collar workers to be covered by a smokefree policy; these differences were statistically significant $(\mathrm{P}<0.0001)$. Conversely, service and blue-collar workers were more likely than white-collar workers to report that their workplace policy allowed smoking in some or all areas of the workplace.
Reported workplace smoking policy coverage also varied by the smoking status of the respondent, with smokers consistently reporting less restrictive coverage than non-smokers. Overall, slightly more than a third of all smokers surveyed reported that they worked under a smoke-free policy, whereas nearly a half of all non-smokers reported that they had a smoke-free policy in their workplaces. Among white-collar employees, smokers were $10 \%$ less likely than non-smokers to report the presence of a policy that did not permit smoking $(\mathbf{P}<0.0001$; table 3$)$. The differences were consistent for service and blue-collar smokers, but they were not as large, averaging about seven percentage points. Less than half the white-collar non-smokers were working under either no policy or policies that allowed smoking in the workplace, whereas more than $60 \%$ of non-smoking service workers and more than $70 \%$ of non-smoking blue-collar workers reported working under these conditions in 1992-93.

Table 3 American workers aged 15+ reporting various smoking policies in the workplace, by occupational category and smoking status

\begin{tabular}{|c|c|c|c|c|c|c|c|c|}
\hline & $\begin{array}{l}\text { Smoke-free } \\
(\%)\end{array}$ & $(95 \% C I)$ & $\begin{array}{l}\text { No policy } \\
(\%)\end{array}$ & $(95 \% C I)$ & $\begin{array}{l}\text { No smoking, work } \\
\text { areas only } \\
\text { (\%) }\end{array}$ & $(95 \% C I)$ & $\begin{array}{l}\text { Smoking } \\
\text { allowed } \\
(\%)\end{array}$ & $(95 \% C I)$ \\
\hline \multicolumn{9}{|l|}{ All workers } \\
\hline Smoker & 36.3 & $(35.6-37.0)$ & 22.3 & $(21.7-22.9)$ & 19.8 & $(19.2-20.4)$ & 21.1 & $(20.5-21.7)$ \\
\hline Non-smoker & 49.1 & $(48.7-49.5)$ & 16.9 & $(16.6-17.2)$ & 18.3 & $(18.0-18.6)$ & 15.7 & $(15.4-16.0)$ \\
\hline All & 46.0 & $(45.6-46.4)$ & 18.4 & $(18.1-18.7)$ & 18.7 & $(18.4-19.0)$ & 17.0 & $(16.7-17.3)$ \\
\hline \multicolumn{9}{|l|}{ White collar } \\
\hline Smoker & 45.2 & $(44.2-46.2)$ & 19.6 & $(18.8-20.4)$ & 17.6 & $(16.8-18.3)$ & 17.6 & $(16.9-18.4)$ \\
\hline Non-smoker & 55.8 & $(55.3-56.3)$ & 14.4 & $(14.0-14.7)$ & 16.4 & $(16.0-16.7)$ & 13.5 & $(13.1-13.8)$ \\
\hline All & 53.7 & $(53.2-54.1)$ & 15.4 & $(15.1-15.8)$ & 16.6 & $(16.3-16.9)$ & 14.3 & $(14.0-14.6)$ \\
\hline \multicolumn{9}{|l|}{ Service } \\
\hline Smoker & 29.8 & $(28.1-31.4)$ & 24.6 & $(23.1-26.1)$ & 21.1 & $(19.6-22.6)$ & 24.5 & $(23.0-26.1)$ \\
\hline Non-smoker & 37.2 & $(36.0-38.4)$ & 21.6 & $(20.5-22.6)$ & 20.4 & $(19.5-21.4)$ & 20.8 & $(19.8-21.8)$ \\
\hline All & 34.8 & $(34.4-35.3)$ & 22.5 & $(22.2-22.9)$ & 20.7 & $(20.3-21.0)$ & 22.0 & $(21.2-22.8)$ \\
\hline \multicolumn{9}{|l|}{ Blue collar } \\
\hline Smoker & 23.0 & $(21.8-24.1)$ & 27.5 & $(26.2-28.8)$ & 23.6 & $(22.4-24.8)$ & 25.9 & $(24.7-27.2)$ \\
\hline Non-smoker & 29.8 & $(28.9-30.8)$ & 23.9 & $(23.0-24.9)$ & 25.0 & $(24.1-25.9)$ & 21.3 & $(20.4-22.1)$ \\
\hline All & 27.4 & $(26.7-28.2)$ & 25.2 & $(24.4-25.9)$ & 24.5 & $(23.8-25.2)$ & 22.9 & $(22.2-23.6)$ \\
\hline
\end{tabular}

$\mathrm{CI}=$ confidence intervals. 
Table 4 American workers covered by smoke-free policies and their smoking prevalence, by occupational category

\begin{tabular}{|c|c|c|c|c|c|}
\hline $\begin{array}{l}\text { Standard Census Bureau Occupational } \\
\text { Categories (codes) (number of interviews) }\end{array}$ & $\begin{array}{l}\text { Smoke-free } \\
(\%)\end{array}$ & $(95 \% C I)$ & $\begin{array}{l}\text { Smoking } \\
\text { prevalence } \\
(\%)\end{array}$ & $(95 \% C I)$ & $\begin{array}{l}\text { Workers in } \\
\text { occupationf (n) }\end{array}$ \\
\hline $\begin{array}{l}\text { Food service occupations } \\
(433-444)(n=5957)\end{array}$ & 21.1 & $(19.9-22.3)$ & 34.3 & $(32.9-35.7)$ & 5516086 \\
\hline $\begin{array}{l}\text { Other transport occupations and } \\
\text { material moving }\end{array}$ & & & & & \\
\hline$(823-859)(\mathrm{n}=508)$ & 22.6 & $(18.4-26.8)$ & 37.8 & $(32.9-42.7)$ & 1137081 \\
\hline $\begin{array}{l}\text { Construction trades } \\
(553-599)(n=908)\end{array}$ & & $(19.8-26.1)$ & 32.9 & $(29.4-36.4)$ & 3485288 \\
\hline Fabricators/assemblers/inspectors/samplers & 22.9 & $(19.0-20.1)$ & & $(29.4-30.4)$ & \\
\hline $\begin{array}{l}(783-799)(n=2475) \\
\text { Machine operators/tenders, excluding }\end{array}$ & 26.3 & $(24.3-28.3)$ & 33.7 & $(31.5-35.9)$ & 2514753 \\
\hline $\begin{array}{l}\text { precision }(703-779)(\mathrm{n}=4988) \\
\text { Mechanics and repairers }\end{array}$ & 26.7 & $(25.3-28.2)$ & 36.3 & $(34.7-37.9)$ & 4642883 \\
\hline$(503-549)(n=2824)$ & 27.0 & $(25.1-28.9)$ & 35.5 & $(33.4-37.6)$ & 3798910 \\
\hline $\begin{array}{l}\text { Other handlers/equipment cleaners/labourers } \\
(864-868 ; 874 ; 885-889)(\mathrm{n}=1484)\end{array}$ & 27.0 & $(24.4-29.6)$ & 38.7 & $(35.8-41.6)$ & 2040942 \\
\hline $\begin{array}{l}\text { Motor vehicle operators } \\
\quad(803-814)(\mathrm{n}=358)\end{array}$ & & & & $(2.0-41.0)$ & \\
\hline $\begin{array}{l}(803-814)(\mathrm{n}=358) \\
\text { Other precision production occupations }\end{array}$ & 28.9 & $(23.5-34.3)$ & 33.4 & $(27.8-39.0)$ & 3432109 \\
\hline $\begin{array}{l}(613-699)(\mathrm{n}=3598) \\
\text { Farm workers and related occupations }\end{array}$ & 30.0 & $(28.2-31.8)$ & 33.6 & $(31.8-35.4)$ & 3597173 \\
\hline $\begin{array}{l}\quad(477-489)(\mathrm{n}=304) \\
\text { Freight/stock/material handlers }\end{array}$ & 30.0 & $(24.0-36.0)$ & 32.0 & $(25.9-38.1)$ & 1770210 \\
\hline $\begin{array}{l}(875-883)(n=1540) \\
\text { Protective service occupations }\end{array}$ & 31.6 & $(29.0-34.3)$ & 30.2 & $(27.6-32.8)$ & 1781065 \\
\hline$(413-427)(n=1237)$ & 36.2 & $(33.2-39.2)$ & 27.5 & $(24.7-30.3)$ & 2109396 \\
\hline $\begin{array}{l}\text { Sales workers, retail/personal services } \\
(263-278)(\mathrm{n}=5726)\end{array}$ & 37.8 & $(36.3-39.3)$ & 25.3 & $(24.0-26.7)$ & 5592229 \\
\hline $\begin{array}{l}\text { Sales representatives, commodities, } \\
\text { excluding retail } \\
(258-259)(\mathrm{n}=738)\end{array}$ & & & & & \\
\hline $\begin{array}{l}(258-259)(n=738) \\
\text { Supervisors and proprietors, sales }\end{array}$ & 38.5 & $(34.4-42.6)$ & 22.4 & $(18.9-25.9)$ & 1346188 \\
\hline $\begin{array}{l}(243)(n=2860) \\
\text { Cleaning/building service occupations }\end{array}$ & 40.7 & $(38.6-42.8)$ & 27.2 & $(25.3-29.1)$ & 2633311 \\
\hline $\begin{array}{l}(448-455)(n=2367) \\
\text { Lawyers and judges }\end{array}$ & 41.6 & $(39.3-43.9)$ & 32.0 & $(29.8-34.2)$ & 2870642 \\
\hline$(178-179)(n=550)$ & 44.6 & $(39.6-49.6)$ & 8.4 & $(5.6-11.2)$ & 445305 \\
\hline $\begin{array}{l}\text { Financial records, processing occupations } \\
(337-344)(\mathrm{n}=2511)\end{array}$ & 45.7 & $(43.3-48.1)$ & 25.1 & $(23.0-27.2)$ & 1979999 \\
\hline $\begin{array}{l}\text { Personal services occupations } \\
(456-469)(\mathrm{n}=1598) \\
\text { Other executive, administrators, managers }\end{array}$ & 47.8 & $(44.8-50.8)$ & 27.3 & $(24.6-30.0)$ & 1743150 \\
\hline $\begin{array}{l}\text { Other executive, administrators, managers } \\
(007-022)(\mathrm{n}=9109)\end{array}$ & 48.2 & $(47.0-49.4)$ & 21.0 & $(20.0-22.0)$ & 8075918 \\
\hline $\begin{array}{l}\text { Mail and message distributing } \\
(354-357)(\mathrm{n}=640)\end{array}$ & 48.2 & $(43.5-52.9)$ & 26.2 & $(22.1-30.3)$ & 919716 \\
\hline $\begin{array}{l}\text { Engineering and science technicians } \\
(213-225)(\mathrm{n}=1124)\end{array}$ & 48.7 & $(45.3-52.1)$ & 24.0 & $(21.1-26.9)$ & 1076204 \\
\hline $\begin{array}{l}\text { Sales representatives, finance, and } \\
\text { business service }(253-257)(n=1370)\end{array}$ & 50.0 & $(46.9-53.1)$ & 20.2 & $(17.7-22.7)$ & 1634928 \\
\hline $\begin{array}{l}\text { Computer equipment operators } \\
(308-309)(\mathrm{n}=708)\end{array}$ & 50.9 & $(46.6-55.2)$ & 28.4 & $(24.5-32.3)$ & 628340 \\
\hline $\begin{array}{l}\text { Supervisors-administrative support } \\
\quad(303-307)(\mathrm{n}=885)\end{array}$ & 51.1 & $(47.2-55.0)$ & 23.4 & $(20.1-26.7)$ & 771350 \\
\hline $\begin{array}{l}\text { Engineers }(044-059)(\mathrm{n}=1874) \\
\text { Management-related occupations }\end{array}$ & 51.6 & $(49.0-54.2)$ & 13.5 & $(11.7-15.3)$ & 1714158 \\
\hline $\begin{array}{l}(023-037)(n=4202) \\
\text { Other administrative support occupations, } \\
\text { including clerical }\end{array}$ & 52.5 & $(50.7-54.3)$ & 16.6 & $(15.3-18.0)$ & 3653579 \\
\hline $\begin{array}{l}(316-336 ; 345-353 ; 359-389)(n=10897) \\
\text { Secretaries, stenographers, typists }\end{array}$ & 53.9 & $(52.8-55.0)$ & 24.3 & $(23.3-25.3)$ & 9398830 \\
\hline$(313-315)(\mathrm{n}=5132)$ & 53.9 & $(52.2-55.6)$ & 21.5 & $(20.1-22.9)$ & 4096948 \\
\hline $\begin{array}{l}\text { Technicians, other }(226-235)(n=1608) \\
\text { Health service occupations }\end{array}$ & 54.3 & $(51.4-57.2)$ & 20.2 & $(17.9-22.5)$ & 1447771 \\
\hline $\begin{array}{l}(445-447)(\mathrm{n}=2181) \\
\text { Administrators/officials, public administration }\end{array}$ & 55.4 & $(52.9-57.9)$ & 31.6 & $(29.2-34.0)$ & 2113638 \\
\hline $\begin{array}{l}(003-006)(n=812) \\
\text { Other professional specialty occupations }\end{array}$ & 57.5 & $(53.4-61.6)$ & 21.3 & $(17.9-24.7)$ & 674509 \\
\hline $\begin{array}{l}(043 ; 063 ; 163-177 ; 183-199)(n=3605) \\
\text { Math/computer scientists }\end{array}$ & 58.2 & $(56.3-60.2)$ & 16.4 & $(14.9-17.9)$ & 3155911 \\
\hline $\begin{array}{l}(064-068)(n=1074) \\
\text { Teachers, college and university }\end{array}$ & 59.8 & $(56.5-63.1)$ & 15.2 & $(12.8-17.6)$ & 927700 \\
\hline$(113-154)(\mathrm{n}=1031)$ & 60.3 & $(56.5-64.1)$ & 9.8 & $(7.5-12.1)$ & 731468 \\
\hline $\begin{array}{l}\text { Natural scientists }(069-083)(n=557) \\
\text { Teachers, excluding college and university }\end{array}$ & 68.9 & $(64.2-73.6)$ & 12.0 & $(8.7-15.3)$ & 477368 \\
\hline $\begin{array}{l}(155-159)(n=5590) \\
\text { Health technologists and technicians }\end{array}$ & 70.4 & $(68.9-71.9)$ & 10.3 & $(9.3-11.3)$ & 4304867 \\
\hline $\begin{array}{l}(203-208)(n=1817) \\
\text { Health assessment/treating occupations }\end{array}$ & 72.4 & $(69.9-74.9)$ & 21.3 & $(19.0-23.6)$ & 1487788 \\
\hline $\begin{array}{l}\quad(095-106)(n=3117) \\
\text { Health diagnosing occupations }\end{array}$ & 76.8 & $(74.9-78.7)$ & 16.8 & $(15.2-18.5)$ & 2478306 \\
\hline$(084-089)(n=428)$ & 80.7 & $(76.3-85.1)$ & 6.7 & $(3.9-9.5)$ & 387247 \\
\hline
\end{tabular}

${ }^{\star}$ Estimates based only on the responses obtained from workers who met the indoor worker criteria (see Method).

t Number of American workers in each occupational category estimated using all persons who responded to the CPS labour force core questionnaire that they were currently working (worked in the week before interview). Self-employed workers not included. 


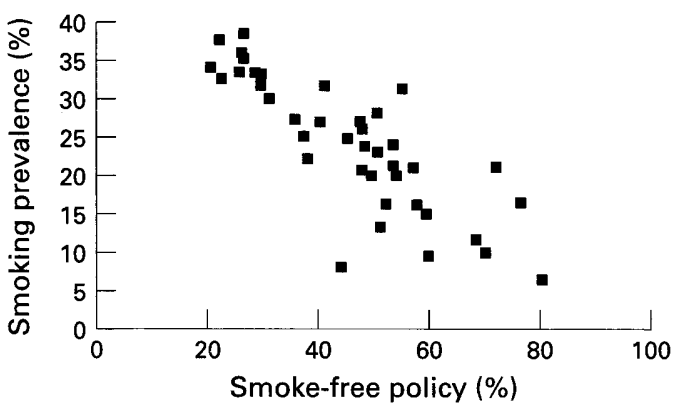

Correlation of the prevalence of smoking and the presence of smoke-free workplace policies for 40 occupational groups. (The graph represents data from table $4(\mathrm{r}=-0.8216$, $P<0.0001)$.)

To examine policy differences by specific occupational classification, each worker was placed in one of the 45 standard Census Bureau occupational groups. Five of the 45 groups are predominantly outdoor occupations-for example, forestry and fishing, construction labourer-and, after applying the indoor worker exclusion criteria, very few workers $(n<100)$ remained in these groups. Responses from workers in these five groups were, therefore, eliminated from further analyses. The 40 remaining groups were ranked in ascending order by the likelihood of the workers in those groups being covered by a smoke-free policy (table 4). The number of currently employed workers in each occupation as well as their smoking prevalence was also determined.

The variation in responses across the occupational groups was substantial. The difference between the highest and lowest ranked occupations was fourfold. The occupational groups with the highest reported rates of coverage were those related to health and healthcare. Workers in health diagnosing occupations (physicians, dentists, veterinarians, optometrists, and podiatrists) reported the highest coverage; $80.7 \%$ reported smoke-free places of employment. The complete reverse was found for workers employed in food service occupations (wait staff, cooks, bartenders, and counter help), only $21.1 \%$ of whom were covered by a smoke-free policy. Moreover, food service workers were $24.9 \%$ less likely than all other workers $(P<0.0001)$ and $13.5 \%$ less likely than other service sector workers to report working under a smoke-free policy $(\mathrm{P}<0.0001)$.

Smoking prevalence also varied by occupation, with health diagnosing occupations having a $6.7 \%$ smoking rate and handlers/equipment cleaners/labourers having a $38.7 \%$ rate. Among the 40 occupational groups, there was a strong negative relationship between smoking prevalence and having a smoke-free policy (figure; $r=-0.8216$, $\mathrm{P}<0.0001$ ).

\section{Discussion}

Protecting workers in the workplace through policy-based smoking restrictions is an issue that has gained considerable attention in the past few years. Previous studies designed to measure the extent of workplace smoking restrictions have not routinely analysed policies from the perspective of the worker. Between 1986 and 1991 the Bureau of National Affairs conducted three studies based on a small sample of active members of the Society of Human Resource Management. ${ }^{13-15}$ These reports showed a steady increase in the number of businesses with smoking policies. Of the businesses covered by this survey, $85 \%$ had instituted policies and $34 \%$ reported smoke-free policies by 1991 . The Administrative Management Society Foundation, which has conducted a small, annual Smoking Policy Survey since 1980 , found that the number of companies with smoking policies increased from $16 \%$ in 1980 to $60 \%$ in 1988 . A quarter of the companies responding in 1988 reported smoke-free establishments. ${ }^{16}$

The Office of Disease Prevention and Health Promotion (ODPHP) conducted two nationally representative surveys of businesses in 1985 and $1992 .{ }^{17-19}$ The most recent ODPHP results showed $58.8 \%$ of worksites to be smoke-free, which this survey defined as a combination of not allowing smoking anywhere inside (34.2\%) and not allowing smoking except in separately ventilated areas $(24.6 \%)$. As part of its Community Intervention Trial for Smoking Cessation (COMMIT), ${ }^{20}$ the NCI also collected data from businesses concerning workplace smoking policies. $^{21}$ In the 1990 survey, which included only companies with 50 or more employees, $15 \%$ of company representatives reported having smoke-free workplaces. ${ }^{22}$ The 1993 survey included businesses that employed as few as 25 employees and found that $44 \%$ of them had implemented smoke-free policies. $^{21}$

Adults in the COMMIT communities were also surveyed, and in 1989 , fewer than $20 \%$ of those who were employed reported working under a smoke-free policy. In 1993, nearly $40 \%$ of workers in the COMMIT survey had smoke-free policies in their workplaces. ${ }^{21}$ The only other national survey of adults to examine this issue before the CPS Tobacco Use Supplement was the 1986 Adult Use of Tobacco Survey. The results from this survey indicated that $45 \%$ of employed adults had some smoking restrictions at their place of work, and only $3 \%$ reported having a smoke-free work environment. ${ }^{23}$

Although these studies provide some measure of the types of smoking restrictions in American workplaces, only two reported restrictions from the perspective of the worker, and only one of these was designed to be nationally representative. The data from the 1992-93 CPS Tobacco Use Supplement, which characterise workplace smoking policies reported by a nationally representative sample of American workers, improve on previous reports in various ways. Whereas the emphasis of the CPS is on providing labour force statistics for use by the United States government, the large number of workers interviewed should constitute a sample representative of all American workers with respect to variables such as race, age, gender, and geographical 
area. Another strength of this survey over some others is that it queried individual workers about workplace policies, in contrast to other surveys, which asked a representative to respond for the entire workforce. Because such respondents were often workers at the managerial level, their perspective concerning smoking policies in the workplace might differ considerably from the other workers in that business. For example, the data from the COMMIT communities showed that individual workers reported rates of smoke-free workplaces that were four to five percentage points lower than those reported by management-level representatives. ${ }^{21}$

Compared with the $3 \%$ of workers nationally who in 1986 reported a "no smoking" policy, the results from personal interviews with workers for the CPS Tobacco Use Supplement demonstrated notable improvements in these policies for most sectors of the US workforce. Nevertheless, most (54.1\%) of American workers were still not covered by a smoke-free workplace policy in 1992-93. Fully $50 \%$ of all non-smokers were not covered by smoke-free policies, and the reports of service and blue-collar non-smokers showed even lower rates of coverage.

The segment of the workforce for whom the prevalence of smoke-free workplaces was lowest was workers aged 15-19. Only $32.1 \%$ of workers in this age group reported that their workplace had implemented a smoke-free policy. This finding may reflect the fact that $43.9 \%$ of currently employed teenagers in this survey worked in service sector jobs (food service and retail sales) that had relatively weak workplace smoking policies. In 1991, Wasserman and colleagues described a potential impact of smoke-free work environments on teenagers and reported that, in comparison to cigarette tax increases, restricting smoking in workplaces had a stronger negative impact on teenage cigarette consumption. ${ }^{24}$

Rates of smoke-free workplace policies for workers in occupations requiring less education or less advanced skills were lower than for workers in occupations requiring college, advanced degrees, or high levels of skill (see table 4). A clear example of the disparity in the provision of smoke-free workplace policies can be seen by examining the policies reported by workers in the food service industry. Workers in this occupational group were the least likely to work under a policy prohibiting smoking in both work and public areas. Nearly $79 \%$ of food service workers reported that they were covered by no policy or by a policy that permitted some smoking in the workplace.

Although the goal should be to provide policies that protect all workers from ETS, the hospitality industry, which employs many food service workers, is often exempted from legislation designed to achieve this goal. ${ }^{25}$ In the past, legislators at all levels of government have hesitated to include bars and restaurants in regulations to protect workers from ETS and have instead focused on the protection of patrons only. Ignoring these establishments, which are the workplaces for 5.5 million food service workers $(21.8 \%$ of whom are -1 teenagers), endangers the health of many peo- $\frac{0}{\sigma}$ ple. In a recent study, Siegel reported that, even $ᄋ$ after controlling for active smoking, food service workers had a $50 \%$ higher risk of lung can- $\underline{\text { ․ }}$ cer than the general population. ${ }^{26}$ This elevated $\vec{\Rightarrow}$ risk was attributed to exposure to higher levels $\stackrel{\circ}{\rightarrow}$ of ETS, which were determined to be nearly two times higher in restaurants than in office work environments.

Analysis of responses to the Tobacco Use Supplement showed that smokers were less के likely than non-smokers to report smoking $\overrightarrow{0}$

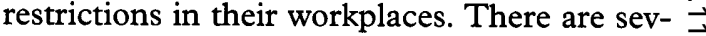
eral possible explanations for this finding, all of $\omega_{\tilde{\sigma}}$ which may be contributing to the overall $\vec{D}$ difference between smokers' and non-smokers' responses. Some of the differential may be due $\omega$ to response bias, with smokers under-reporting $\vec{\bullet}$ smoking restrictions and/or non-smokers overreporting such restrictions. Although smokers were less likely than non-smokers in this survey $c$ to answer "Don't know" when asked whether their workplace had a smoking policy $(1.0 \%$ vs $2.3 \%$ ), indicating that smokers may be more aware of smoking policies in their workplaces than non-smokers, smokers still may have been less likely to report more stringent policies than non-smokers.

Another possible explanation for this reporting difference is that smokers and non-smokers chose their workplaces based on the smoking policies in those workplaces. Although this could be a factor, it is not likely to be a significant contributor to the overall differences observed. In 1991, the average amount of time a worker had spent with his employer was 4.5 years. ${ }^{27}$ Therefore, most workers in this survey would have been in their present jobs during the five years before this survey, and, during that time period, large numbers of workplaces were only beginning to consider smoking policies. ${ }^{15}{ }^{23}$ Additionally, Sorensen et al. found that, at least among smaller companies, only $4 \%$ reported that any employees had left the company due to the implementation of a smoking policy, ${ }^{28}$ which suggests that smoking policies are probably not a determining factor in where a person chooses to work.

Inasmuch as many workplace policies, especially smoke-free provisions, were not dictated by state or local laws in $1992-93,{ }^{18}$ the policies reported in this survey are largely a reflection of self-imposed restrictions. Therefore, another probable explanation for the differences between smokers' and non-smokers' responses is that those workplaces employing relatively higher numbers of smokers were less likely than other workplaces to implement stringent smoking rules. The strong negative correlation between smoking prevalence and smoke-free policies (see figure) lends support to this explanation. Nevertheless, the possibility cannot be disregarded that workers in occupations with more smokers may have reported fewer smoking restrictions based simply on their knowledge of and exposure to greater numbers of smokers while at work and not based on an actual policy in place in their work environment. 
A final explanation for the differences between smokers' and non-smokers' responses to the question that asked about smoking policies is that smoke-free workplaces may have influenced smokers to quit smoking, which would have the effect of increasing the percentage of non-smokers working in smoke-free workplaces. Some studies have shown that smoke-free workplace policies can affect smoking behaviour, including cessation. ${ }^{29}{ }^{30}$

The data provided by the CPS Tobacco Use Supplement indicate that $\mathbf{5 7 . 8}$ million currently employed indoor workers (40.4 million of whom are non-smokers) are not covered by a smoke-free workplace policy and are, therefore, at risk of workplace exposure to a known carcinogen. Recognising the importance of protecting these workers from the demonstrated hazards of ETS, the US Occupational Safety and Health Administration (OSHA) proposed regulations in 1994 that would require employers to provide either a smoke-free workplace or separately ventilated rooms for smokers' use. ${ }^{31}$ In contrast to the vastly unequal policies documented in this report, this rule would provide equal protection to American workers regardless of age, gender, race, or occupation. It is clear from the findings presented here that a large number of American workers would benefit greatly from implementation of the regulations proposed by OSHA.

Note: The 1992-93 CPS Tobacco Use Supplement is available as three public-use data tapes. Those interested should contact the US Bureau of the Census, Customer Service, at +1 301457 4100 (tel) or +3014573842 (fax).

The authors gratefully acknowledge the staff of the US Bureau of the Census who worked on the 1992-93 CPS Tobacco Use Supplement. Special recognition is due Ronald Tucker, Doreen Trammell, and Gregory Weyland for their invaluable assistance in the design, implementation, and processing of the in the design,

1 Steinfeld JL. The public's responsibility: a bill of rights for the non-smoker. RI Med f 1972;55:124-6, 138 .

2 Steinfeld J. Combating smoking in the United States: progress through science and social action. $\mathcal{F}$ Natl Cancer Inst 1991;83:1126-7.

3 US Department of Health, Education and Welfare. Public exposure to air pollution from tobacco smoke. Chapter 8 in: The health consequences of smoking. A report of the Surgeon General, 1972. Washington, DC: National Clearinghouse for Smoking and Health, 1972. (DHEW Publication No (HSM) 72-7516.)

4 US Department of Health and Human Services. The health consequences of involuntary smoking. A report of the Surgeon
General, 1986. Rockville, Maryland: Public Health Service, General, 1986. Rockville, Maryland: Public Health Service, Centers for Disease Con

5 National Research Council. Environmental tobacco smoke: measuring exposures and assessing health effects. Washington, DC: National Academy Press, 1986.

6 US Environmental Protection Agency. Respiratory health effects of passive smoking: lung cancer and other disorders. Smoking and Tobacco Control Monograph No 4 Smoking and Tobacco Control Monograph No 4. Bethesda, Maryland: US Department of Health and tutes of Health, 1993. (NIH Publication No 93-3605.)
7 US Department of Health and Human Services. Healthy people 2000: national health promotion and disease prevention abjectives. Washington, DC: US Government Printing Office, 1990. (DHHS Publication No (PHS) 91-50212.)

8 US Centers for Disease Control and Prevention. Cigarette smoking among adults-United States, 1992, and changes in the definition of current cigarette smoking. MMWR in the definition

9 Hansen RH. The Current Population Survey: design and methodology. Technical Paper No 40. Washington, DC: US Department of Commerce, Bureau of the Census, July 1985.

10 Marcus AC, Crane LA, Shopland DR, Lynn WR. Prevalence of cigarette smoking in the United States. Estimates from the Current Population Survey. 7 Natl Cancer Inst 1989;81:409-14.

11 US Department of Commerce. Current Population Survey, Tobacco Use Supplement. Technical documentation. Washington, DC: Bureau of the Census, 1994.

12 SAS Institute. SAS user's guide. Version 6.11. Cary, North Carolina.

13 Bureau of National Affairs. Where there's smoke: problems and policies concerning smoking in the workplace. Washington, DC: Bureau of National Affairs, 1986

14 Bureau of National Affairs. Where there's smoke: problems and policies concerning smoking in the workplace, 2nd ed. policies concerning smoking in the workplace, 2nd

15 Bureau of National Affairs. Smoking in the workplace, 1991. Society of Human Resource Management-Bureau of National Affairs Survey No 55. Washington, DC: Bureau of National Affairs, 1991.

16 Administrative Management Society. 9th Annual Smoking Policies Survey. Employee Relations Weekly 1989;7:370.

17 Fielding JE. Worksite health promotion survey: smoking control activities. Prev Med 1990;19:402-13.

18 US Department of Health and Human Services. 1992 National survey of worksite health promotion activities. Washington, DC: Public Health Service, Office of Disease Prevention and Health Promotion, 1992.

19 McGinnis JM. 1992 national survey of worksite health promotion activities: summary. Am $\mathcal{f}$ Health Prom 1993 7:453-64.

20 US Department of Health and Human Services. Community-based interventions for smokers: the COMMIT field experience. Smoking and Tobacco Control Monograph No 6. Bethesda, Maryland: Public Health Service, No 6. Bethesda, Maryland: Public Health Service, 1995. NIH Publication No 95-4028.)

21 Glasgow RE, Sorensen G, Giffen C, Shipley RH, Corbett K Lynn W. Promoting worksite smoking control policies and actions: the Community Intervention Trial for Smokin Cessation (COMMIT) experience. Prev Med 1996, 25:186-94.

22 Glasgow RE, Sorensen G, Corbett K. Worksite smoking control activities: prevalence and related worksite characteristics from the COMMIT study, 1990. Prev Med 1992; 21:688-700.

23 Passive smoking: beliefs, attitudes, and exposures-United States, 1986. MMWR 1988;37:239-41.

24 Wasserman J, Manning WG, Newhouse JP, Winkler JD. The effects of excise and regulations on cigarette smoking. $f$ Health Econ 1991;10:43-64.

25 Prohibition on smoking in an enclosed workplace. Compliance guidelines for the hospitality industry. COMAR 09.12.23 and Chapter 5, Acts of 1995 Maryland Occupational Safety and Health.

26 Siegel $M$. Involuntary smoking in the restaurant workplace. A review of employee exposure and health effects. $\mathscr{f} A M A$ 1993;270:490-3.

27 US Department of Labor, Bureau of Labor Statistics Employee tenure and occupational mobility in the early 1990s. News release 92-386, 26 June 1992.

28 Sorensen G, Rosen A, Pinney J, Rudolph J, Doyle N. Worksite smoking policies in small businesses. F Occup Med 1991;33:980-4.

29 Longo DR, Browson RC, Johnson JC, et al. Hospital smoking bans and employee smoking behavior results of a national survey. $\mathscr{F} A M A$ 1996;275:1252-7.

30 Woodruff TJ, Rosbrook B, Pierce J, Glantz SA. Lower level of cigarette consumption found in smoke-free workplaces in California. Arch Intern Med 1993;153:1485-93.

31 US Department of Labor, Occupational Safety and Health Administration. Indoor air quality proposed rule. Part II 29 CFR Parts 1910,1915, 1926 and 1928. Federal Register 1994;59:15968-16039. 